\title{
Oncogeriatrics (part 4.) \\ Pre-operative assessment of elderly patients with cancer
}

\author{
Jakub Kenig
}

Department of General, Oncological and Geriatric Surgery, Jagiellonian University Medical College, Krakow, Poland

Older patients comprise a highly heterogeneous group, and chronological age, comorbidities, or the type of surgical procedure performed cannot adequately describe the risk of adverse post-operative outcomes. Therefore, current routine pre-operative assessment also cannot adequately identify patients at risk. The Comprehensive Geriatric Assessment, the mean life expectancy and the treatment goals of a patient must be included in the pre-operative evaluation. The Comprehensive Geriatric Assessment helps to determine the primary status of an older patient, to diagnose frailty syndrome and to identify how to optimize a patient's condition before surgery. Surgery is one of the primary triggers for disability in older patients. In this age group, being independent is more important than prolonging life. This is particularly true in patients with frailty syndrome, or decreased physiological reserves, which arise from cumulative deficits in several physiological systems and result in a diminished resistance to stressors. Therefore, a standardized pre-operative diagnostic approach, individualized surgical technique selection and tailored post-operative care are essential for successful treatment of elderly patients.

NOWOTWORY J Oncol 2020; 70, 1: 16-19

Key words: older patient, pre-operative assessment, comprehensive geriatric assessment

In geriatric surgery, the key element is to recognize that an elderly patient is not simply an "older adult", and that routine procedures reserved for younger adults may not bring the expected outcomes. In this age group, it is essential to standardize pre-operative diagnostic processes and to personalize treatment and post-operative care. A key factor in geriatric care is the pre-operative assessment, which should be extensive and consider all changes associated with both physiological and pathological ageing. The surgical procedure technique, itself, does not currently differ extensively from accepted standards. However, an increasing number of institutions are introducing modifications to the existing oncological guidelines, especially for patients with frailty syndrome. In addition, there is enough evidence that elderly cancer patients benefit when they undergo minimally invasive surgical and/or endoscopic procedures performed by experienced professionals $[1,2]$. The wide availability of various surgical platforms and techniques provides an unpre- cedented opportunity to offer elderly patients alternative possibilities for their surgeries.

This necessity for personalized care arises from the substantial heterogeneity within the ageing population. Chronological and biological age are different, and with increasing age, these differences become more pronounced. The speed of ageing is unique to individuals and may even differ in separate organs and systems of a single patient. Thus, it is a grave mistake to make decisions on the extent and method of surgery for an elderly patient based solely on chronological age, medical history, physical examination, basic biochemical and imaging tests, as well as consultations. An increasing number of studies show that the surgeon's assessments for surgery eligibility are largely subjective. For example, a large proportion of surgeons associate frailty syndrome with multiple morbidities and/or disability. Fried et al. studied the relationship between these factors and concluded that only $21.5 \%$ of patients were diagnosed with multiple morbidities, disability and frailty syndrome 
and most patients had only one of these factors. They found that $6 \%$ of frailty syndrome patients were disabled (defined as dependency in daily activities on others), while $46 \%$ had multiple morbidities (defined as the presence of two or more accompanying illnesses). The most important observation from this study was that $27 \%$ of patients with frailty syndrome were not disabled and did not have multiple morbidities at all [3].

Routine examinations often lead to a discontinuation of therapy in patients who may potentially qualify for such treatments, and conversely, to seemingly healthy patients being qualified for extensive surgical procedures. Commonly, older patients are disqualified from radical treatments based on an information card containing a long list of diagnoses. However, it may turn out that none of these diseases are a marker illness, and they may not have a significant effect on the post-operative period. However, the opposite may also be true, where a patient reports for surgery without any comorbidities and with documentation showing no counter-indications for surgery. Nonetheless, this patient also has not been adequately assessed prior to the surgical procedure.

Another key aspect in the pre-operative assessment in elderly patients is to define the treatment goal. For young adults, the aim is to achieve all elements, such as curing the illness, preventing complications, alleviating symptoms, prolonging life, maintaining an appropriate level of physical activity, and improving the quality of life. For older patients, the situation is not as obvious. Studies indicate that ensuring a suitable quality of life is significantly more important than prolonging it and maintaining independence in the post-operative period should be a priority [4]. These facets to the treatment goals are also increasingly being noticed by scientists studying cancer. Five-year survival, disease- and complication-free survival are no longer the only endpoints in assessing elderly cancer patients. Other factors that are increasingly being discussed are those that are critical for elderly patients, such as quality of life in the post-operative period and return to pre-operative physical and mental capacity. We need to be aware that often the doctor's goals in designing the treatment do not align with the patient's goals. Additional factors that may prove helpful in better understanding the patient's expectations and what can be offered to them, include: Comprehensive Geriatric Assessment (CGA), knowledge of the remaining average lifespan relative to physical health, and defining the short- and long-term goals of the patient. A study is currently being conducted which aims to answer some basic questions involved in the process of returning to health following surgery:

- How long does an elderly patient need to return to everyday activity?

- How long will they be reliant on others for care?

- What will their mental capacity be after treatment? Knowing the answers to these questions will allow elderly patients to be offered more personalized treatment plans and will allow the patients to give informed consent to such plans. A patient's perception of their own health, mood, and ability to cope with their illness also varies by individual and with time. The same goal does not always suit everyone. A healthy, elderly person will have different goals than a bedridden patient with frailty syndrome. For the first, a key outcome will be staying active, while for the second, it will be alleviating symptoms and the possibility of independently going about their daily activities.

Comprehensive Geriatric Assessment (CGA) is a multidirectional, integrated diagnostic process whose goal is to establish the extent of impairment of welfare [5]. A detailed review of CGA is beyond the scope of this article, so only the most basic information necessary for understanding the process will be discussed here. CGA is a set of diagnostic tools that evaluate everyday:

- functionality,

- physical fitness,

- level of nutrition,

- existing comorbidities,

- risk of depression,

- cognitive function,

- polypharmacotherapy,

- social support.

Its goals are to assess the baseline condition of the patient, identify previously unknown health issues, and diagnose frailty syndrome. This in turn leads to pre-operative "optimization" of the patient's state and may be useful in selecting a treatment strategy. It is estimated that CGA allows for the identification of previously unidentified health issues in up to $40 \%$ of older patients qualifying for surgical treatment [6]. Studies in many different specialties have shown that frailty syndrome is an independent risk factor for poor treatment outcomes in elderly patients [7-9].

Studies conducted at our department showed that a deficit-accumulation model was the most beneficial model for pre-operative patient assessment. The sum of the diagnostic tools, and not their separate individual results, was an independent risk factor of 30-day mortality and post-operative complications. Additionally, the number and type of assessment tools employed had a great effect on how frequently frailty syndrome was identified. A CGA consisting of diagnostic tools measuring functionality, physical and cognitive capacity, levels of depression, level of nutrition, polypharmacotherapy and comorbidities turned out to be the most precise measure predicting post-operative complications and mortality [10].

A large obstacle to the widespread use of CGA is that it requires experience, it is time-consuming, and it is not necessary for all elderly patients. However, in terms of the time consumption, devoting an additional 40-60 minutes to a patient prior to surgery may result in a decreased risk of complications and decreased dependence on others, and could allow the patient to return to physical and cognitive fitness sooner. Financially, there are benefits as well. The cost of care 
frequently multiplies when complications occur and could cause the patient to require prolonged dependence on others and/or being moved to a nursing/caring facility.

An alternative to using the full CGA may be to use a screening study. The current literature contains at least a dozen diagnostic tools dedicated to this. In our studies, we compared six of the most commonly used tools. The abbreviated CGA (aCGA) and the G8 test were the best tools for elderly patients with a cancer diagnosis who were qualified for a surgical procedure; the G8 showed the highest sensitivity and negative predictive value, while the aCGA was better for general assessment [11]. On the other hand, for ad hoc procedures, the best screening test was the Vulnerable Elderly Survey 13 (VES-13). It had the highest sensitivity and negative predictive value in assessing the risk of complications and mortality during the post-operative period. While these approaches require further study, they can already offer clinicians additional information that may be used for post-operative treatment optimization of high-risk elderly patients [12]. One should be aware that screening tools aim to merely identify patients requiring additional geriatric assessments, and they do not allow for reliable identification of problems in individual domains, which in turn prevents planning the appropriate pre-rehabilitation. These tools also have variable efficacy in different populations, which is why it is recommended to analyze the potential usefulness according to one's needs.

Another important consideration to pre-operative assessment in the elderly is that surgical procedures are the single greatest risk factor for disability and dependence on others for care, especially in patients with frailty syndrome. It is therefore worthwhile to briefly discuss the legal aspects. Often, elderly cancer patients are offered a standardized treatment model geared toward younger adults by their doctors, who do so from the fear of being accused of incorrect oncological treatment. In this context, it may be useful to surgeons to highlight the Supreme Court verdict from September 24, 2015 (V CSK $738 / 14$ - the extent of obligation to provide information by doctors), discussed in the article by Dr. Radosław Drozda from the Department of Forensic Medicine at the Wrocław Medical University [13]. It concluded that"the choice between alternative treatment methods belongs to the patient, and the clinician should present the patient with all available treatment options that are possible in their physical condition - at most with an indication as to which of these options is the most beneficial according to the doctor..."and "... it is the patient - despite a lack of medical training - who should make the decision on the surgical method that they will be subjected to. The role of the doctor is to convince the patient why (and for what medical reasons) it would be worth to undergo a riskier procedure. The patient however has the right (driven by personal reasons or even superstition) to pick a method that would be less invasive and is likely to have a lower efficacy than the method proposed by the clinician" [13].
Currently, evidence-based medical decision-making in this age group is met with great difficulty. This is caused in part by the large number of low- and medium-quality publications and in part by the dearth of scientific evidence. The best example of this is a study by Schiphorst et al., which analyzed the involvement of elderly patients in studies of laparoscopic surgeries, performed due to colorectal cancer. As highlighted by the authors, in $85 \%$ of the cases the average age was below 65 years old, and $44 \%$ of studies excluded elderly patients [14]. Extrapolating results from studies conducted on younger patients is a significant error. However, in analyzing the number of new publications devoted to the topic of elderly patients, one can hope that many questions will be answered by increasingly well-designed studies.

In conclusion, in order to improve treatment outcomes, it is necessary to consider issues specific to older populations in the pre-operative patient assessment. The questions presented below can help in this decision-making process:

- Is the currently planned treatment strategy correct? Are there alternative treatment options?

- What is the result of the Comprehensive Geriatric Assessment? Can frailty syndrome be diagnosed in the patient?

- What is the risk of complications?

- What would be the patient's lifespan without treatment?

- What are the goals, preferences and expectations of the patient? What effect might the treatment have on these goals?

- Is it possible to improve the patient's condition prior to the surgical procedure?

\section{Conflict of interest: none declared}

\section{Jakub Kenig}

Jagiellonian University Medical College

Department of General, Oncological and Geriatric Surgery

ul. Pradnicka 35-37

31-202 Kraków, Poland

e-mail:jkenig@cm-uj.krakow.pl

\section{Received and accepted: 16 Dec 2019}

\section{References}

1. Antoniou SA, Antoniou GA, Koch OO, et al. Laparoscopic colorectal surgery confers lower mortality in the elderly: a systematic review and meta-analysis of 66,483 patients. Surg Endosc. 2015; 29(2):322-333, doi: 10.1007/s00464-014-3672-x, indexed in Pubmed: 24986017.

2. Sun V, Fong Y. Minimally Invasive Cancer Surgery: Indications and Outcomes. Semin Oncol Nurs. 2017; 33(1): 23-36, doi: 10.1016/j. soncn.2016.11.003, indexed in Pubmed: 28062330.

3. Fried LP, Tangen CM, Walston J, et al. Cardiovascular Health Study Collaborative Research Group. Frailty in older adults: evidence for a phenotype. J Gerontol A Biol Sci Med Sci. 2001; 56(3): M146-M156, doi: 10.1093/gerona/56.3.m146, indexed in Pubmed: 11253156.

4. Deiner S, Silverstein JH. Long-term outcomes in elderly surgical patients Mt Sinai J Med. 2012; 79(1): 95-106, doi: 10.1002/msj.21288, indexed in Pubmed: 22238042.

5. Ramani L, Furmedge DS, Reddy SPH. Comprehensive geriatric assessment. Br J Hosp Med (Lond). 2014; 75 Suppl 8: C122-C125, doi: 10.12968/hmed.2014.75.sup8.c122, indexed in Pubmed: 25237688. 
6. Ellis G, Whitehead MA, O'Neill D, et al. Comprehensive geriatric assessment for older adults admitted to hospital. Cochrane Database Syst Rev. 2011(7): CD006211, doi: 10.1002/14651858.CD006211.pub2, indexed in Pubmed: 21735403.

7. Makary MA, Segev DL, Pronovost PJ, et al. Frailty as a predictor of surgical outcomes in older patients. J Am Coll Surg. 2010; 210(6): 901-908, doi: 10.1016/j.jamcollsurg.2010.01.028, indexed in Pubmed: 20510798.

8. Arya S, Kim SIn, Duwayri Y, et al. Frailty increases the risk of 30-day mortality, morbidity, and failure to rescue after elective abdominal aortic aneurysm repair independent of age and comorbidities. J Vasc Surg. 2015; 61(2): 324-331, doi: 10.1016/j.jvs.2014.08.115, indexed in Pubmed: 25312534

9. Hall DE, Arya S, Schmid KK, et al. Association of a Frailty Screening Initiative With Postoperative Survival at 30, 180, and 365 Days. JAMA Surg. 2017; 152(3): 233-240, doi: 10.1001/jamasurg.2016.4219, indexed in Pubmed: 27902826.

10. Kenig J, Zychiewicz B, Olszewska U, et al. Screening for frailty among older patients with cancer that qualify for abdominal surgery. J Geriatr
Oncol. 2015; 6(1): 52-59, doi: 10.1016/j.jgo.2014.09.179, indexed in Pubmed: 25277874.

11. Kenig J, Olszewska U, Zychiewicz B, et al. Cumulative deficit model of geriatric assessment to predict the postoperative outcomes of older patients with solid abdominal cancer. J Geriatr Oncol. 2015; 6(5): 370-379, doi: 10.1016/j.jgo.2015.03.004, indexed in Pubmed: 26144556.

12. Kenig J, Zychiewicz B, Olszewska $U$, et al. Six screening instruments for frailty in older patients qualified for emergency abdominal surgery. Arch Gerontol Geriatr. 2015; 61(3): 437-442, doi: 10.1016/j. archger.2015.06.018, indexed in Pubmed: 26211706.

13. Drozd R. Kto powinien wybierać metodę leczenia operacyjnego - pacjent czy lekarz? Chirurgia po Dyplomie. 2017; 2(12): 34-36.

14. Schiphorst AHW, Pronk A, Borel Rinkes IHM, et al. Representation of the elderly in trials of laparoscopic surgery for colorectal cancer. Colorectal Dis. 2014; 16(12): 976-983, doi: 10.1111/codi.12806, indexed in Pubmed: 25331635. 